Western North American Naturalist 68(2), (C) 2008, pp. 173-185

\title{
AQUATIC INVERTEBRATE FAUNA OF A NORTHERN PRAIRIE STREAM: RANGE EXTENSIONS AND WATER QUALITY CHARACTERISTICS
}

\author{
Iain D. Phillips ${ }^{1}$, Dale Parker $^{2}$, and Glen McMaster ${ }^{3}$
}

\begin{abstract}
The benthic macroinvertebrate fauna of southern Saskatchewan, Canada, has received little attention relative to other regions of western North America. Therefore, little is known of the related aquatic ecosystem health and biogeography of regional aquatic insects. Here we present the results of an aquatic macroinvertebrate survey for the Pipestone Creek watershed in southeastern Saskatchewan. We qualitatively sampled aquatic macroinvertebrates in 5 sites on 4 dates through spring, summer, and fall 2006. Sampling produced 294 taxa of macroinvertebrates including 25 provincial range extensions to the southeast corner of the province. Presence/absence data of taxa grouped the sites into lentic and lotic sites. However, the relative proportions of the taxa varied greatly among sites, with no 2 sites having a community similarity greater than $50 \%$. Functional feeding group analyses separated the sites into collector-dominated and scraper/grazer-dominated sites. However, the taxonomic make-up of the feeding groups varied among sites and also among dates. A modified Hilsenhoff Biotic Index of the site communities indicated that all were influenced by organic pollution. Results of this study suggest that although the watershed is enormously diverse, its biological communities are likely influenced by organic pollution. Further, range expansions of species found here, such as the stonefly Perlesta placida, have implications for invasion pathways and post-glaciation species islands in a prairie landscape.
\end{abstract}

Key words: benthic macroinvertebrates, southeast Saskatchewan, Canada, Pipestone Creek, range extensions.

Over 1500 taxa of aquatic macroinvertebrates have been recorded from Saskatchewan. These macroinvertebrates are a significant part of the province's biodiversity and an essential component of its aquatic ecosystems. Representatives occupy every functional feeding group and play a critical role in recycling organic materials back into the food chain (Resh and Rosenberg 1984, Thorp and Covich 2001). Many are extremely important in the diets of game fish, waterfowl, and passerine birds. Others are pests, and some, such as Culex tarsalis, transmit diseases (e.g., West Nile virus) to humans and animals. Still others, such as several large-river mayflies, are important conservation topics (e.g., in the South Saskatchewan River), as they are at risk of extinction (Webb 2002).

Most research involving aquatic macroinvertebrates in Saskatchewan has been related to monitoring the environmental impacts of mining, logging, and other developments on aquatic ecosystems. These studies traditionally involved replicated control/treatment sampling and density measures. Unfortunately, the vast majority of this research has been conducted in specific locations rather than broader geographic areas such as watersheds or at coarser taxonomic levels; therefore, we have an opportunity to contribute detailed biodiversity knowledge to this area of North America.

Pipestone Creek is an important source of water for many communities and farms in southeastern Saskatchewan. A number of game fish, waterfowl species, and deer inhabit the watershed, providing recreation and food to the area's residents. The creek and its wetlands also provide breeding habitat for biting flies, which are not only a nuisance but can transmit diseases. The primary objective of our research was to begin documenting aquatic macroinvertebrate biodiversity and assemblages found in the Pipestone Creek watershed in southeastern Saskatchewan. In addition, by assessing the macroinvertebrate assemblage structure at evenly spaced distances along the length of Pipestone Creek and its associated tributaries, we were able to identify areas of potentially degraded water quality that may be contributing to the river as it leaves the province. Through metrics such as species richness and the Hilsenhoff Biotic Index

\footnotetext{
${ }^{1}$ Stewardship Division, Saskatchewan Watershed Authority, \#330-350 3rd Avenue North, Saskatoon, SK, Canada S7K 2H6. E-mail: iain.phillips@swa.ca

2 AquaTax Consulting, 1204 Main Street, Saskatoon, SK, Canada S7H 0L2.

${ }^{3}$ Stewardship Division, Saskatchewan Watershed Authority, Suite 420-2365 Albert Street, Regina, SK, Canada S4P 4K1.
} 


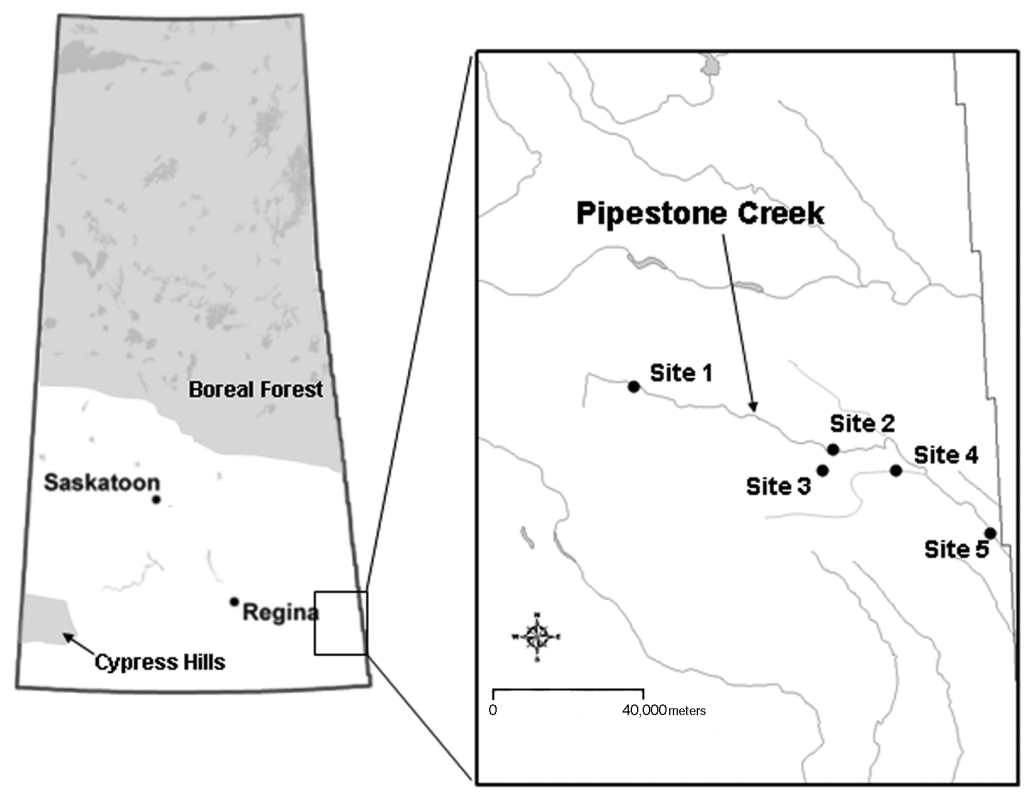

Fig. 2. Cluster analysis of site taxa presence/absence data.

TABLE 1. Average water chemistry values for Pipestone Creek sampling sites, April-September 2006. Standard errors are in parentheses. Sample sizes are as follows: $n=4$ for sites $1,2,4$, and $5 ; n=3$ for site 3 .

\begin{tabular}{lccccc}
\hline & \multicolumn{5}{c}{ Study site } \\
\cline { 2 - 6 } Parameter & Site 1 & Site 2 & Site 3 & Site 4 & Site 5 \\
\hline pH & $8.02(0.04)$ & $8.24(0.03)$ & $8.27(0.12)$ & $8.20(0.09)$ & $8.32(0.04)$ \\
Dissolved solids $(\mathrm{ppm})$ & $455.75(11.06)$ & $731.50(39.96)$ & $765.00(45.61)$ & $905.25(28.65)$ & $695.00(21.03)$ \\
Conductivity $(\mu \mathrm{S})$ & $756.5(18.37)$ & $1205(62.62)$ & $1276.50(76.54)$ & $1514.50(47.37)$ & $1160.25(35.08)$ \\
Temperature $\left({ }^{\circ} \mathrm{C}\right)$ & $17.75(1.79)$ & $20.53(1.85)$ & $20.95(5.41)$ & $18.78(1.04)$ & $18.90(1.34)$ \\
Turbidity & Low & High & Low & Low & High \\
\hline
\end{tabular}

between sites in lotic reaches of the watershed, we attempt to identify sites that may be valuable to conserve and on which to restrict further agricultural development.

\section{Study SiTES}

Pipestone Creek drains an area of $1340 \mathrm{~km}^{2}$ of the Aspen Parkland Ecoregion in the southeastern corner of Saskatchewan (Fig. 1). It is a low-gradient stream that meanders along the bottom of a glacial spillway known as the Pipestone Spillway, which drained Lake Indian Head about 12,700 years BP. The creek eventually flows into Manitoba, enters Oak Lake, and ultimately becomes part of the Souris River system. Numerous permanent and intermittent streams, oxbow lakes, beaver ponds, and wetlands are associated with Pipestone Creek along its course. Two man-made dams on the creek have formed Pipestone Lake and Moosomin Lake. During spring runoff and periods of high precipitation, flow rates can be swift in undammed portions of the channel, but during late summer or drought conditions the channel may become dry.

All sites are slightly alkaline, and all but site 1 overlapped in dissolved solids and conductivity through the course of the sampling period (Table 1). The sites are generally similar to each other in riparian zone vegetation and damage caused by cattle grazing and watering. Chief differences among the sites are water flow, water level changes, macrophytes, and turbidity. Sites 1 and 4 are clear and very slow flowing, with extensive areas of submersed and emergent vegetation, and relatively stable water levels. Site 1 has lower dissolved solids and 
conductivity than the other sites. At site 3 the water was clear and slow flowing except at the culvert and has extensive amounts of submersed and emergent vegetation, but surface water disappeared from the sampling area by August. Water at sites 2 and 5 was very turbid and fast flowing initially, but by August it became pools and trickles due to water loss. Submersed and emergent vegetation was sparse at both sites throughout the year and silt coated most surfaces.

\section{Specific Site Descriptions}

SITE $1\left(50^{\circ} 15^{\prime} 12^{\prime \prime} \mathrm{N}, 102^{\circ} 37^{\prime} 42^{\prime \prime} \mathrm{W}\right)$. - At this site a trail and bridge artificially create an openwater wetland with an approximately 50/50 split of open water and emergent vegetation, mainly Typha spp. The substrate in the open water is submersed vegetation over gravel and silt. The substrate in the emergent vegetation area is a decaying vegetation mat over a predominantly silt substrate. Initially, on 25 April 2006, there was moderate water flow over parts of the gravel trail (water depth $20-30 \mathrm{~cm}$ ) and under the bridge (water depth $70 \mathrm{~cm}$ ). However, beavers dammed these areas and maintained the dam throughout the summer and fall. This essentially stopped water flow and stabilized the water level behind the dam. Downstream the creek is further obstructed by a gravel road, which forces the creek through culverts. Cattle grazing and drinking heavily impact the riparian zone at the site. Willows are the dominant shrubs and small clumps of aspen are present.

SITE $2\left(50^{\circ} 07^{\prime} 47^{\prime \prime} \mathrm{N}, 101^{\circ} 58^{\prime} 39^{\prime \prime} \mathrm{W}\right)$.- Pipestone Creek at site 2 flows through a steepsided artificial channel constructed to improve water flow through a bridged gravel road crossing downstream from the collection site. Water current was moderately rapid in spring and early summer. However, water levels declined by late summer to such an extent that water flow was reduced to small trickles between pools. The substrate is silt in depositional areas and moderate cobble and gravel in scour stretches. The water is very turbid and all submersed substrates, including macrophytes, are covered by silt. Mid-channel water depths in spring were $1.6 \mathrm{~m}$ or greater in the pools.

SITE $3\left(50^{\circ} 04^{\prime} 33^{\prime \prime} \mathrm{N}, 101^{\circ} 56^{\prime} 11^{\prime \prime} \mathrm{W}\right)$. - This unnamed intermittent creek flows into Pipestone Creek during spring and high-precipitation periods. By late summer the stream was reduced to pools, which dried for the remainder of the year. The substrate consists of fine organic silt with decaying vegetation. Emergent vegetation in the ditch includes Typha, sedges, and grasses. Submerged vegetation is mostly Utricularia.

Site $4\left(50^{\circ} 02^{\prime} 02^{\prime \prime} \mathrm{N}, 101^{\circ} 41^{\prime} 41^{\prime \prime} \mathrm{W}\right)$.-This site on Little Pipestone Creek flows into wetlands associated with Moosomin Lake less than $2 \mathrm{~km}$ from Moosomin Dam. The creek channel is steep sided and up to $1.8 \mathrm{~m}$ deep and $5.0 \mathrm{~m}$ across. The water is clear except when cattle disturb the substrate. The bottom substrate is silt intermixed with decaying vegetation debris. Vegetation is abundant, with Typha being the most common emergent. Submerged vegetation includes Utricularia, Rannuculus, and Myriophyllum. Shore vegetation includes sedges and a variety of shrubs and grasses.

Site $5\left(49^{\circ} 53^{\prime} 11^{\prime \prime} \mathrm{N}, 101^{\circ} 26^{\prime} 57^{\prime \prime} \mathrm{W}\right)$.- - This site is on the main stem of Pipestone Creek at a bridged gravel road crossing approximately $40 \mathrm{~km}$ downstream from the Moosomin Dam. During spring the creek is fast flowing. However, flow rates are dependent on the amount of water released at the dam. Water flow declined dramatically through the summer up until August, when only trickles and pools were present at the site. At the sampling site the substrate is large cobble with sand and silt in sheltered areas.

\section{METHODS}

Sampling was conducted during 4 periods in 2006: 25 April, 29 and 30 May, 5 and 6 July, and 14, 15, and 16 August. Site 3 was completely sampled only on 29 May and 5 July.

Using a Hanna HI98129 "Combo" pH and EC water meter, we measured basic water parameters (temperature, $\mathrm{pH}$, dissolved solids, and conductivity) at each site. Current velocity and turbidity were described qualitatively.

At each site we used a 1-mm-mesh, Dframed aquatic net and a 750- $\mu \mathrm{m}$ sieve to qualitatively sample all microhabitat types that could be accessed in chest waders. Submerged rocks, logs, etc., were examined for adhering macroinvertebrates. In addition, a composite proportional dip net sample was taken either as a kick sample, if current velocity and water depth allowed, or as a series of jabs and sweeps of the substrate and submerged vegetation. These samples were preserved in the field 
TABLE 2. Macroinvertebrate taxa identified from 5 study sites in Pipestone Creek and its tributaries.

\begin{tabular}{|c|c|c|c|c|c|}
\hline Major taxa & Site 1 & Site 2 & Site 3 & Site 4 & Site 5 \\
\hline TOTAL COUNT $=294$ & 143 & 116 & 99 & 159 & 153 \\
\hline \multicolumn{6}{|l|}{ PORIFERA } \\
\hline \multicolumn{6}{|l|}{ Spongillidae } \\
\hline Ephydatia fluviatilis Linnaeus & & & $\mathrm{X}$ & $\mathrm{X}$ & \\
\hline Plumatella repens (Linnaeus) & $\mathrm{X}$ & & & & 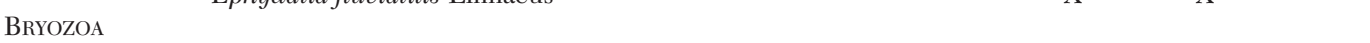 \\
\hline \multicolumn{6}{|l|}{ ANNELIDA } \\
\hline \multicolumn{6}{|l|}{ Clitellata: Hirudinea } \\
\hline \multicolumn{6}{|l|}{ Erpobdellidae } \\
\hline Erpobdella punctata Sawyer \& Shelley & $\mathrm{X}$ & & & $\mathrm{X}$ & $\mathrm{X}$ \\
\hline Nephelopsis obscura Verrill & $\mathrm{X}$ & & $\mathrm{X}$ & & \\
\hline \multicolumn{6}{|l|}{ Glossiphoniidae } \\
\hline Glossiphonia complanata (Linnaeus) & $\mathrm{X}$ & & & $\mathrm{X}$ & $\mathrm{X}$ \\
\hline Helobdella fusca (Castle) & & & $\mathrm{X}$ & & \\
\hline Helobdella stagnalis Linnaeus & $\mathrm{X}$ & & & $\mathrm{X}$ & \\
\hline Placobdella ornata (Verrill) & $\mathrm{X}$ & & $\mathrm{X}$ & & $\mathrm{X}$ \\
\hline Theromyzon spp. & & & & $\mathrm{X}$ & \\
\hline \multicolumn{6}{|l|}{ MollusCa } \\
\hline \multicolumn{6}{|l|}{ Gastropoda } \\
\hline \multicolumn{6}{|l|}{ Ancylidae } \\
\hline Ferrissia rivularis (Say) & & $\mathrm{X}$ & & & $\mathrm{X}$ \\
\hline \multicolumn{6}{|l|}{ Lymnaeidae } \\
\hline Lymnaea stagnalis (Linnaeus) & $\mathrm{X}$ & & $\mathrm{X}$ & $\mathrm{X}$ & \\
\hline Pseudosuccinea columella (Say) & $\mathrm{X}$ & & $\mathrm{X}$ & $\mathrm{X}$ & $\mathrm{X}$ \\
\hline Stagnicola elodes (Say) & $\mathrm{X}$ & & $\mathrm{X}$ & $\mathrm{X}$ & \\
\hline Stagnicola exilis (I. Lea) & $\mathrm{X}$ & & $\mathrm{X}$ & $\mathrm{X}$ & \\
\hline \multicolumn{6}{|l|}{ Physidae } \\
\hline Physa gyrina $($ Say $)$ & & $\mathrm{X}$ & & $\mathrm{X}$ & $\mathrm{X}$ \\
\hline Physa skinneri Taylor & $\mathrm{X}$ & $\mathrm{X}$ & $\mathrm{X}$ & $\mathrm{X}$ & \\
\hline \multicolumn{6}{|l|}{ Planorbidae } \\
\hline Armiger crista (Linnaeus) & $\mathrm{X}$ & & & $\mathrm{X}$ & \\
\hline Gyraulus circumstriatus (Tryon) & $\mathrm{X}$ & & $\mathrm{X}$ & $\mathrm{X}$ & \\
\hline Gyraulus deflectus (Say) & & & $\mathrm{X}$ & $\mathrm{X}$ & \\
\hline Helisoma anceps anceps (Menke) & & & & & $\mathrm{X}$ \\
\hline Helisoma pilsbryi infracarinatum (Baker) & & & & $\mathrm{X}$ & \\
\hline Planorbella subcrenata (Carpenter) & $\mathrm{X}$ & $\mathrm{X}$ & $\mathrm{X}$ & & \\
\hline \multirow{2}{*}{\multicolumn{6}{|c|}{ Valvatidae }} \\
\hline & & & & & \\
\hline Valvata tricarinata $($ Say) & & $\mathrm{X}$ & & $\mathrm{X}$ & $\mathrm{X}$ \\
\hline \multirow{2}{*}{\multicolumn{6}{|c|}{$\begin{array}{l}\text { Pelecypoda } \\
\text { Pisidiidae }\end{array}$}} \\
\hline Pisidiidae & & & & & \\
\hline Pisidium spp. & $\mathrm{X}$ & $\mathrm{X}$ & & $\mathrm{X}$ & $\mathrm{X}$ \\
\hline Sphaerium spp. & & $\mathrm{X}$ & & $\mathrm{X}$ & $\mathrm{X}$ \\
\hline Unionidae & & & & & \\
\hline Pyganodon grandis (Say) & & $\mathrm{X}$ & & & $\mathrm{X}$ \\
\hline ARTHRopoda: CRUSTACEA & & & & & \\
\hline Amphipoda (immatures) & $\mathrm{X}$ & $\mathrm{X}$ & $\mathrm{X}$ & $\mathrm{X}$ & $\mathrm{X}$ \\
\hline Gammaridae & & & & & \\
\hline Gammarus lacustris Sars & $\mathrm{X}$ & & & $\mathrm{X}$ & $\mathrm{X}$ \\
\hline Hyallelidae & & & & & \\
\hline Hyallela azteca Saussure & $\mathrm{X}$ & $\mathrm{X}$ & $\mathrm{X}$ & $\mathrm{X}$ & $\mathrm{X}$ \\
\hline Decapoda & & & & & \\
\hline Cambaridae & & & & & \\
\hline Orconectes virilis Hagen & & $\mathrm{X}$ & & & $\mathrm{X}$ \\
\hline Ostracoda spp. & $\mathrm{X}$ & $\mathrm{X}$ & $\mathrm{X}$ & $\mathrm{X}$ & $\mathrm{X}$ \\
\hline ARTHROPODA: CHELICERATA & & & & & \\
\hline Arachnida: Acari & & & & & \\
\hline Acari spp. & $\mathrm{X}$ & $\mathrm{X}$ & $\mathrm{X}$ & $\mathrm{X}$ & $\mathrm{X}$ \\
\hline ARTHROPODA: INSECTA & & & & & \\
\hline Ephemeroptera & & & & & \\
\hline Baetidae & & & & & \\
\hline Acerpenna pygmaea (Hagen) & & & & & $\mathrm{X}$ \\
\hline
\end{tabular}


Table 2. Continued.

\begin{tabular}{|c|c|c|c|c|c|}
\hline Major taxa & Site 1 & Site 2 & Site 3 & Site 4 & Site 5 \\
\hline Baetis brunneicolor McDunnough & & & & $\mathrm{X}$ & $\mathrm{X}$ \\
\hline Baetis flavistriga McDunnough & & $\mathrm{X}$ & & $\mathrm{X}$ & $\mathrm{X}$ \\
\hline Baetis sp. C & & & & & $\mathrm{X}$ \\
\hline Baetis sp. 03 & & $\mathrm{X}$ & & & \\
\hline Baetis spp. & & $\mathrm{X}$ & & & \\
\hline Baetis tricaudatus/brunneicolor & & & & & $\mathrm{X}$ \\
\hline Callibaetis ferrugineus (Walsh) & & $\mathrm{X}$ & $\mathrm{X}$ & $\mathrm{X}$ & $\mathrm{X}$ \\
\hline Callibaetis pallidus Banks & & & & & $\mathrm{X}$ \\
\hline Procloeon spp. & & $\mathrm{X}$ & $\mathrm{X}$ & & \\
\hline \multicolumn{6}{|l|}{ Caenidae } \\
\hline Caenis spp. & $\mathrm{X}$ & $\mathrm{X}$ & $\mathrm{X}$ & $\mathrm{X}$ & $\mathrm{X}$ \\
\hline Caenis amica Hagen & $\mathrm{X}$ & $\mathrm{X}$ & $\mathrm{X}$ & $\mathrm{X}$ & \\
\hline Caenis latipennis Banks & $\mathrm{X}$ & $\mathrm{X}$ & $\mathrm{X}$ & $\mathrm{X}$ & $\mathrm{X}$ \\
\hline Caenis youngi Roemhild & $\mathrm{X}$ & $\mathrm{X}$ & $\mathrm{X}$ & $\mathrm{X}$ & $\mathrm{X}$ \\
\hline \multicolumn{6}{|l|}{ Ephemeridae } \\
\hline Hexagenia limbata $(\mathrm{Hex})$ & & $\mathrm{X}$ & & & $\mathrm{X}$ \\
\hline \multicolumn{6}{|l|}{ Heptageniidae } \\
\hline \multicolumn{6}{|l|}{ Leucrocuta hebe (McDunnough) } \\
\hline Stenacron interpunctatum (Say) & & $\mathrm{X}$ & $\mathrm{X}$ & & $\mathrm{X}$ \\
\hline \multirow{2}{*}{\multicolumn{6}{|c|}{ Leptophlebiidae }} \\
\hline & & & & & \\
\hline Leptophlebia spp. & & & & & $\mathrm{X}$ \\
\hline \multicolumn{6}{|l|}{ Odonata } \\
\hline \multicolumn{6}{|l|}{ Calopterygidae } \\
\hline Calopteryx aequabilis Say & & $\mathrm{X}$ & & & \\
\hline \multicolumn{6}{|l|}{ Coenagrionidae } \\
\hline Coenagrion angulatum Walker & & & & $\mathrm{X}$ & \\
\hline Coenagrion resolutum (Hagen) & $\mathrm{X}$ & & & $\mathrm{X}$ & \\
\hline Enallagma boreale (Selys) & & & & $\mathrm{X}$ & \\
\hline Enallagma cyathigerum (Charpentier) & $\mathrm{X}$ & & & & $\mathrm{X}$ \\
\hline Enallagma ebrium (Hagen) & $\mathrm{X}$ & $\mathrm{X}$ & $\mathrm{X}$ & $\mathrm{X}$ & $\mathrm{X}$ \\
\hline Enallagma vernale Gloyd & & & & $\mathrm{X}$ & \\
\hline Enallagma/Coenagrion spp. & $\mathrm{X}$ & $\mathrm{X}$ & $\mathrm{X}$ & $\mathrm{X}$ & $\mathrm{X}$ \\
\hline Nehalennia irene (Selys) & & & $\mathrm{X}$ & & \\
\hline \multicolumn{6}{|l|}{ Lestidae } \\
\hline Lestes spp. & & & $\mathrm{X}$ & $\mathrm{X}$ & $\mathrm{X}$ \\
\hline Lestes congener Hagen & $\mathrm{X}$ & $\mathrm{X}$ & & $\mathrm{X}$ & $\mathrm{X}$ \\
\hline Lestes disjunctus Selys & $\mathrm{X}$ & $\mathrm{X}$ & & & $\mathrm{X}$ \\
\hline Lestes unguiculatus Hagen & $\mathrm{X}$ & $\mathrm{X}$ & $\mathrm{X}$ & & $\mathrm{X}$ \\
\hline \multicolumn{6}{|l|}{ Aeshnidae } \\
\hline Aeshna interrupta Walker & $\mathrm{X}$ & $\mathrm{X}$ & & $\mathrm{X}$ & \\
\hline Aeshna umbrosa Walker & & & & & $\mathrm{X}$ \\
\hline Anax junius (Drury) & & & & $\mathrm{X}$ & \\
\hline \multicolumn{6}{|l|}{ Cordulidae } \\
\hline Tetragoneuria canis MacLachlan & & & & $\mathrm{X}$ & \\
\hline \multicolumn{6}{|l|}{ Libellulidae } \\
\hline Lecorrhinia spp. & $\mathrm{X}$ & & & & \\
\hline Leucorrhinia intacta (Hagen) & $\mathrm{X}$ & & & & \\
\hline Leucorrhinia patricia Walker & $\mathrm{X}$ & & & & \\
\hline Leucorrhinia proxima Calvert & $\mathrm{X}$ & & & & \\
\hline Libellula quadrimaculata Linnaeus & $\mathrm{X}$ & & & $\mathrm{X}$ & $\mathrm{X}$ \\
\hline Somatochlora spp. & & $\mathrm{X}$ & & & \\
\hline Somatochlora ensigera Martin & & $\mathrm{X}$ & & & \\
\hline Sympetrum spp. & $\mathrm{X}$ & & & & \\
\hline Sympetrum costiferum (Hagen) & $\mathrm{X}$ & $\mathrm{X}$ & $\mathrm{X}$ & & $\mathrm{X}$ \\
\hline Sympetrum internum Montgomery & $\mathrm{X}$ & $\mathrm{X}$ & $\mathrm{X}$ & $\mathrm{X}$ & $\mathrm{X}$ \\
\hline Sympetrum obtrusum (Hagen) & $\mathrm{X}$ & & & & \\
\hline \multirow{2}{*}{\multicolumn{6}{|c|}{$\begin{array}{l}\text { Plecoptera } \\
\text { Perlidae }\end{array}$}} \\
\hline & & & & & \\
\hline Perlesta placida (Hagen) & & $\mathrm{X}$ & $\mathrm{X}$ & & $\mathrm{X}$ \\
\hline Hemiptera & & & & & \\
\hline $\begin{array}{l}\text { Belostomatidae } \\
\text { Lethocerus americanus (Leidy) }\end{array}$ & $\mathrm{X}$ & & & $\mathrm{X}$ & \\
\hline
\end{tabular}


Table 2. Continued.

\begin{tabular}{|c|c|c|c|c|c|}
\hline Major taxa & Site 1 & Site 2 & Site 3 & Site 4 & Site 5 \\
\hline \multicolumn{6}{|l|}{ Corixidae } \\
\hline Callicorixa audeni Hungerford & $\mathrm{X}$ & $\mathrm{X}$ & $\mathrm{X}$ & $\mathrm{X}$ & $\mathrm{X}$ \\
\hline Coenocorixa dakotensis (Hungerford) & & & & & $\mathrm{X}$ \\
\hline Cymatia americana Hussey & $\mathrm{X}$ & & & $\mathrm{X}$ & \\
\hline Hesperocorixa spp. & $\mathrm{X}$ & & & $\mathrm{X}$ & $\mathrm{X}$ \\
\hline Hesperocorixa atopodonta (Hungerford) & & & & $\mathrm{X}$ & \\
\hline Hesperocorixa michiganesis (Hungerford) & $\mathrm{X}$ & & & & \\
\hline Hesperocorixa vulgaris (Hungerford) & $\mathrm{X}$ & & & $\mathrm{X}$ & \\
\hline Palmacorixa gillettei Abbott & & & & & $\mathrm{X}$ \\
\hline Sigara alternata (Say) & & $\mathrm{X}$ & & & $\mathrm{X}$ \\
\hline Sigara bicolorpennis (Walley) & $\mathrm{X}$ & & & $\mathrm{X}$ & $\mathrm{X}$ \\
\hline Sigara grossolineata Hungerford & $\mathrm{X}$ & $\mathrm{X}$ & & $\mathrm{X}$ & $\mathrm{X}$ \\
\hline Sigara lineata (Forster) & & $\mathrm{X}$ & & & $\mathrm{X}$ \\
\hline Sigara solensis (Hungerford) & & & & $\mathrm{X}$ & \\
\hline Trichocorixa borealis Sailer & & & & & $\mathrm{X}$ \\
\hline Trichocorixa naias (Kirkaldy) & $\mathrm{X}$ & $\mathrm{X}$ & & $\mathrm{X}$ & $\mathrm{X}$ \\
\hline \multicolumn{6}{|l|}{ Gerridae } \\
\hline Gerris comatus Drake and Hottes & & $\mathrm{X}$ & & & \\
\hline Gerris buenoi Kirkaldy & $\mathrm{X}$ & $\mathrm{X}$ & & $\mathrm{X}$ & $\mathrm{X}$ \\
\hline Gerris dissortis Drake and Harris & & $\mathrm{X}$ & & & $\mathrm{X}$ \\
\hline \multirow{2}{*}{\multicolumn{6}{|c|}{$\begin{array}{l}\text { Gerris pingreenis Drake and Hottes } \\
\text { Nepidae }\end{array}$}} \\
\hline & & & & & \\
\hline Ranatra fusca Palisot & & & & $\mathrm{X}$ & $\mathrm{X}$ \\
\hline \multicolumn{6}{|l|}{ Notonectidae } \\
\hline Notonecta borealis Hussey & & & $\mathrm{X}$ & $\mathrm{X}$ & $\mathrm{X}$ \\
\hline Notonecta undulata Say & $\mathrm{X}$ & $\mathrm{X}$ & & $\mathrm{X}$ & $\mathrm{X}$ \\
\hline \multicolumn{6}{|l|}{ Veliidae } \\
\hline Microvelia buenoi Drake & $\mathrm{X}$ & & & & \\
\hline \multicolumn{6}{|l|}{ Trichoptera } \\
\hline \multicolumn{6}{|l|}{ Hydropsychidae } \\
\hline Cheumatopsyche spp. & $\mathrm{X}$ & $\mathrm{X}$ & $\mathrm{X}$ & & $\mathrm{X}$ \\
\hline Cheumatopsyche campyla Ross & & & & & $\mathrm{X}$ \\
\hline Hydropsyche alternans (Walker) & & $\mathrm{X}$ & & & $\mathrm{X}$ \\
\hline Hydropsyche bifida Banks & & & & & $\mathrm{X}$ \\
\hline Hydropsyche riola Denning & & & $\mathrm{X}$ & & $\mathrm{X}$ \\
\hline \multicolumn{6}{|l|}{ Hydroptilidae } \\
\hline Hydroptila spp. & $\mathrm{X}$ & $\mathrm{X}$ & $\mathrm{X}$ & $\mathrm{X}$ & $\mathrm{X}$ \\
\hline Ithytrichia clavata Morton & & & & & $\mathrm{X}$ \\
\hline Neotrichia spp. & & & & & $\mathrm{X}$ \\
\hline \multicolumn{6}{|l|}{ Leptoceridae } \\
\hline Ceraclea spp. & & & $\mathrm{X}$ & & \\
\hline Nectopsyche diarina (Ross) & & $\mathrm{X}$ & & & $\mathrm{X}$ \\
\hline Oecetis immobilis (Hagen) & & & $\mathrm{X}$ & $\mathrm{X}$ & \\
\hline Oecetis ochracea (Curtis) & & $\mathrm{X}$ & & & \\
\hline Triaenodes spp. & & & $\mathrm{X}$ & $\mathrm{X}$ & \\
\hline Triaenodes injustus (Hagen) & $\mathrm{X}$ & & & & \\
\hline Triaenodes nox Ross & & & $\mathrm{X}$ & $\mathrm{X}$ & \\
\hline \multicolumn{6}{|l|}{ Limnephilidae } \\
\hline Anabolia bimaculata (Walker) & $\mathrm{X}$ & $\mathrm{X}$ & $\mathrm{X}$ & $\mathrm{X}$ & $\mathrm{X}$ \\
\hline Limnephilus cf. janus Ross & & & & $\mathrm{X}$ & \\
\hline Limnephilus hyalinus Hagen & $\mathrm{X}$ & & & $\mathrm{X}$ & \\
\hline Limnephilus infernalis (Banks) & $\mathrm{X}$ & & $\mathrm{X}$ & $\mathrm{X}$ & \\
\hline Limnephilus labus Ross & & & $\mathrm{X}$ & & \\
\hline Limnephilus rhombicus (Linnaeus) & & & & $\mathrm{X}$ & \\
\hline Philarctus quaeris (Milne) & & & $\mathrm{X}$ & $\mathrm{X}$ & \\
\hline \multicolumn{6}{|l|}{ Phrygaenidae } \\
\hline Agrypnia spp. & $\mathrm{X}$ & & & & \\
\hline Agrypnia straminea Hagen & $\mathrm{X}$ & & & & \\
\hline Phryganea cinerea Walker & & & & $\mathrm{X}$ & \\
\hline Ptilostomis semifasciata (Say) & & & & $\mathrm{X}$ & \\
\hline Ptilostomis spp. & & $\mathrm{X}$ & & & \\
\hline \multicolumn{6}{|l|}{ Polycentropodidae } \\
\hline Neureclipsis spp. & & & & $\mathrm{X}$ & \\
\hline Polycentropus spp. & & $\mathrm{X}$ & & & $\mathrm{X}$ \\
\hline
\end{tabular}


Table 2. Continued.

\begin{tabular}{|c|c|c|c|c|c|}
\hline Major taxa & Site 1 & Site 2 & Site 3 & Site 4 & Site 5 \\
\hline \multicolumn{6}{|l|}{ Lepidoptera } \\
\hline Pyralidae & & $\mathrm{X}$ & $\mathrm{X}$ & & \\
\hline \multicolumn{6}{|l|}{ Megaloptera } \\
\hline \multicolumn{6}{|l|}{ Sialidae } \\
\hline Sialis velata Ross & & & & & $\mathrm{X}$ \\
\hline \multicolumn{6}{|l|}{ Coleoptera } \\
\hline \multicolumn{6}{|l|}{ Dytiscidae } \\
\hline Acilius semisulcatus Aubé & $\mathrm{X}$ & & & $\mathrm{X}$ & \\
\hline Agabus spp. & $\mathrm{X}$ & & $\mathrm{X}$ & $\mathrm{X}$ & \\
\hline Colymbetes exaratus LeConte & & & & $\mathrm{X}$ & \\
\hline Dytiscus spp. & $\mathrm{X}$ & & $\mathrm{X}$ & $\mathrm{X}$ & \\
\hline Graphoderus occidentalis Horn & & & & $\mathrm{X}$ & \\
\hline Graphoderus perplexus Sharp & & & & $\mathrm{X}$ & \\
\hline Hydaticus piceus LeConte & & & & $\mathrm{X}$ & \\
\hline Hydroporus spp. & $\mathrm{X}$ & & & $\mathrm{X}$ & $\mathrm{X}$ \\
\hline Hygrotus sayi J. Balfour-Browne & $\mathrm{X}$ & & $\mathrm{X}$ & $\mathrm{X}$ & \\
\hline Hygrotus sellatus LeConte & $\mathrm{X}$ & & $\mathrm{X}$ & & \\
\hline Ilybius discendens Sharp & & & & & $\mathrm{X}$ \\
\hline Ilybius subaeneus Erichson & & & & $\mathrm{X}$ & \\
\hline Laccophilus spp. & & & & $\mathrm{X}$ & \\
\hline Laccophilus biguttatus Kirby & $\mathrm{X}$ & & & $\mathrm{X}$ & \\
\hline Laccophilus maculosus Say & $\mathrm{X}$ & $\mathrm{X}$ & & & $\mathrm{X}$ \\
\hline Liodessus spp. & & & & $\mathrm{X}$ & \\
\hline Nebrioporus macronychus (Shirt) & & & & & $\mathrm{X}$ \\
\hline Neoporus superioris (J. Balfour-Browne) & $\mathrm{X}$ & & & $\mathrm{X}$ & $\mathrm{X}$ \\
\hline Neoporus undulatus (Say) & $\mathrm{X}$ & & & $\mathrm{X}$ & \\
\hline Neoporus vittatus (LeConte) & & $\mathrm{X}$ & & $\mathrm{X}$ & $\mathrm{X}$ \\
\hline Oreodytes spp. & & & & & $\mathrm{X}$ \\
\hline Rhantus sericans Sharp & & & & $\mathrm{X}$ & \\
\hline Rhantus wallisi Hatch & $\mathrm{X}$ & & & & \\
\hline \multicolumn{6}{|l|}{ Elmidae } \\
\hline Dubiraphia spp. & & $\mathrm{X}$ & $\mathrm{X}$ & $\mathrm{X}$ & $\mathrm{X}$ \\
\hline \multicolumn{6}{|l|}{ Gyrinidae } \\
\hline Gyrinus maculiventris LeConte & & & $\mathrm{X}$ & $\mathrm{X}$ & \\
\hline Gyrinus pectoralis LeConte & & & & $\mathrm{X}$ & \\
\hline \multicolumn{6}{|l|}{ Haliplidae } \\
\hline Haliplus spp. & $\mathrm{X}$ & $\mathrm{X}$ & $\mathrm{X}$ & $\mathrm{X}$ & \\
\hline Haliplus apicalis Thomson & $\mathrm{X}$ & & & $\mathrm{X}$ & \\
\hline Haliplus borealis LeConte & & & & & $\mathrm{X}$ \\
\hline Haliplus immaculicollis Harris & $\mathrm{X}$ & $\mathrm{X}$ & $\mathrm{X}$ & $\mathrm{X}$ & $\mathrm{X}$ \\
\hline Haliplus stagninus Leech & $\mathrm{X}$ & & & & \\
\hline Haliplus subguttatus Roberts & & & $\mathrm{X}$ & & \\
\hline Peltodytes tortulosus Roberts & $\mathrm{X}$ & & & & $\mathrm{X}$ \\
\hline \multicolumn{6}{|l|}{ Hydrophilidae } \\
\hline Berosus spp. & $\mathrm{X}$ & & $\mathrm{X}$ & $\mathrm{X}$ & \\
\hline Berosus striatus (Say) & $\mathrm{X}$ & & & $\mathrm{X}$ & \\
\hline Enochrus diffusus (LeConte) & $\mathrm{X}$ & & & $\mathrm{X}$ & $\mathrm{X}$ \\
\hline Enochrus hamiltoni (Horn) & $\mathrm{X}$ & $\mathrm{X}$ & & $\mathrm{X}$ & $\mathrm{X}$ \\
\hline Helophorus spp. & & $\mathrm{X}$ & & & \\
\hline Hydrobius fuscipes (Linnaeus) & $\mathrm{X}$ & & & & \\
\hline \multicolumn{6}{|l|}{ Diptera } \\
\hline Ceratopogonidae & $\mathrm{X}$ & $\mathrm{X}$ & $\mathrm{X}$ & $\mathrm{X}$ & $\mathrm{X}$ \\
\hline Bezzia/Palpomyia spp. & $\mathrm{X}$ & & $\mathrm{X}$ & & \\
\hline Forcipomyia spp. & & $\mathrm{X}$ & & & \\
\hline \multicolumn{6}{|l|}{ Chaoboridae } \\
\hline Chaoborus flavicens (Meigen) & $\mathrm{X}$ & & & $\mathrm{X}$ & $\mathrm{X}$ \\
\hline \multicolumn{6}{|l|}{ Chironomidae } \\
\hline Tanypodinae & $\mathrm{X}$ & $\mathrm{X}$ & $\mathrm{X}$ & $\mathrm{X}$ & $\mathrm{X}$ \\
\hline Ablabesmyia spp. & $\mathrm{X}$ & & $\mathrm{X}$ & $\mathrm{X}$ & $\mathrm{X}$ \\
\hline Ablabesmyia illinoensis Malloch & $\mathrm{X}$ & & & & \\
\hline Clinotanypus spp. & & & & $\mathrm{X}$ & \\
\hline
\end{tabular}


Table 2. Continued.

\begin{tabular}{|c|c|c|c|c|c|}
\hline Major taxa & Site 1 & Site 2 & Site 3 & Site 4 & Site 5 \\
\hline Conchapelopia spp. & & & & & $\mathrm{X}$ \\
\hline Labrundinia neopilosella Beck \& Beck & $\mathrm{X}$ & & $\mathrm{X}$ & & $\mathrm{X}$ \\
\hline Procladius spp. & $\mathrm{X}$ & $\mathrm{X}$ & $\mathrm{X}$ & $\mathrm{X}$ & $\mathrm{X}$ \\
\hline Tanypus spp. & & & $\mathrm{X}$ & & \\
\hline Thienemannimyia complex & $\mathrm{X}$ & $\mathrm{X}$ & & $\mathrm{X}$ & $\mathrm{X}$ \\
\hline \multicolumn{6}{|l|}{ Chironomidae } \\
\hline \multicolumn{6}{|l|}{ Orthocladiinae } \\
\hline Acricotopus spp. & $\mathrm{X}$ & & & $\mathrm{X}$ & $\mathrm{X}$ \\
\hline Corynoneura spp. & $\mathrm{X}$ & & $\mathrm{X}$ & $\mathrm{X}$ & \\
\hline Cricotopus spp. & $\mathrm{X}$ & $\mathrm{X}$ & $\mathrm{X}$ & $\mathrm{X}$ & $\mathrm{X}$ \\
\hline Cricotopus sylvestris gr. & $\mathrm{X}$ & & $\mathrm{X}$ & $\mathrm{X}$ & \\
\hline Epoicocladius spp. & & $\mathrm{X}$ & & & \\
\hline Eukiefferiella spp. & & & & & $\mathrm{X}$ \\
\hline Orthocladius/Cricotopus spp. & $\mathrm{X}$ & $\mathrm{X}$ & $\mathrm{X}$ & $\mathrm{X}$ & $\mathrm{X}$ \\
\hline Psectrocladius spp. & $\mathrm{X}$ & $\mathrm{X}$ & & $\mathrm{X}$ & $\mathrm{X}$ \\
\hline Reocricotopus spp. & & & & & $\mathrm{X}$ \\
\hline Thienemanniella spp. & & $\mathrm{X}$ & & & \\
\hline Chironominae: Chironomini & $\mathrm{X}$ & $\mathrm{X}$ & $\mathrm{X}$ & $\mathrm{X}$ & $\mathrm{X}$ \\
\hline Chironomus spp. & $\mathrm{X}$ & $\mathrm{X}$ & & $\mathrm{X}$ & $\mathrm{X}$ \\
\hline Cladopelma spp. & $\mathrm{X}$ & & $\mathrm{X}$ & $\mathrm{X}$ & \\
\hline Cryptochironomus spp. & & $\mathrm{X}$ & & & $\mathrm{X}$ \\
\hline Cryptochironomus curryi Mason & & & & & $\mathrm{X}$ \\
\hline Cryptochironomus eminentia Mason & & & & & $\mathrm{X}$ \\
\hline Cryptochironomus sorex Townes & & & & & $\mathrm{X}$ \\
\hline Cryptotendipes spp. & & $\mathrm{X}$ & $\mathrm{X}$ & $\mathrm{X}$ & \\
\hline Dicrotendipes spp. & $\mathrm{X}$ & $\mathrm{X}$ & $\mathrm{X}$ & $\mathrm{X}$ & $\mathrm{X}$ \\
\hline Endochironomus spp. & $\mathrm{X}$ & $\mathrm{X}$ & $\mathrm{X}$ & $\mathrm{X}$ & $\mathrm{X}$ \\
\hline Glyptotendipes spp. & $\mathrm{X}$ & $\mathrm{X}$ & $\mathrm{X}$ & $\mathrm{X}$ & \\
\hline Microtendipes spp. & & $\mathrm{X}$ & & & $\mathrm{X}$ \\
\hline Parachironomus spp. & $\mathrm{X}$ & & $\mathrm{X}$ & $\mathrm{X}$ & \\
\hline Paratendipes spp. & & $\mathrm{X}$ & & & $\mathrm{X}$ \\
\hline Phaenopsectra spp. & & $\mathrm{X}$ & & $\mathrm{X}$ & $\mathrm{X}$ \\
\hline Polypedilum spp. & $\mathrm{X}$ & $\mathrm{X}$ & $\mathrm{X}$ & $\mathrm{X}$ & $\mathrm{X}$ \\
\hline Polypedilum flavum (Johannsen) & & $\mathrm{X}$ & & & $\mathrm{X}$ \\
\hline Stictochironomus spp. & & $\mathrm{X}$ & & & $\mathrm{X}$ \\
\hline Zavreliella marmorata (van der Wulp) & & & & $\mathrm{X}$ & \\
\hline Pseudochironomus spp. & $\mathrm{X}$ & & & & \\
\hline Chironimoninae: Tanytarsini & $\mathrm{X}$ & $\mathrm{X}$ & $\mathrm{X}$ & $\mathrm{X}$ & $\mathrm{X}$ \\
\hline Cladotanytarsus spp. & & $\mathrm{X}$ & & & \\
\hline Paratanytarsus spp. & $\mathrm{X}$ & $\mathrm{X}$ & $\mathrm{X}$ & $\mathrm{X}$ & $\mathrm{X}$ \\
\hline Tanytarsus spp. & $\mathrm{X}$ & & $\mathrm{X}$ & $\mathrm{X}$ & \\
\hline \multicolumn{6}{|l|}{ Culicidae } \\
\hline Aedes spp. & & & & & $\mathrm{X}$ \\
\hline Anopheles earlei Vargas & $\mathrm{X}$ & & $\mathrm{X}$ & $\mathrm{X}$ & \\
\hline \multicolumn{6}{|l|}{ Dixidae } \\
\hline Dixella spp. & & & & $\mathrm{X}$ & $\mathrm{X}$ \\
\hline Dolichopodidae & & $\mathrm{X}$ & & & \\
\hline Dolichopodidae sp. 1 & & & & & $\mathrm{X}$ \\
\hline Dolichopodidae sp. 2 & $\mathrm{X}$ & $\mathrm{X}$ & & & $\mathrm{X}$ \\
\hline Dolichopodidae sp. 3 & & & & & $\mathrm{X}$ \\
\hline \multicolumn{6}{|l|}{ Psychodidae } \\
\hline Pericoma/Telmatoscopus spp. & $\mathrm{X}$ & & & & \\
\hline Sciomyzidae & $\mathrm{X}$ & $\mathrm{X}$ & & $\mathrm{X}$ & $\mathrm{X}$ \\
\hline \multicolumn{6}{|l|}{ Simuliidae } \\
\hline Simulium spp. & & $\mathrm{X}$ & & & $\mathrm{X}$ \\
\hline Simulium bivittatum Malloch & & $\mathrm{X}$ & & & \\
\hline Simulium venustum/verecundum gr. & & $\mathrm{X}$ & $\mathrm{X}$ & $\mathrm{X}$ & $\mathrm{X}$ \\
\hline Simulium vittatum Zetterstedt & & $\mathrm{X}$ & & & $\mathrm{X}$ \\
\hline Stratiomyidae & & & & & \\
\hline Odontomyia spp. & $\mathrm{X}$ & & & & \\
\hline Syrphidae & & $\mathrm{X}$ & & $\mathrm{X}$ & $\mathrm{X}$ \\
\hline Helophilus spp. & & & & & $\mathrm{X}$ \\
\hline
\end{tabular}


Table 2. Continued.

\begin{tabular}{|c|c|c|c|c|c|}
\hline Major taxa & Site 1 & Site 2 & Site 3 & Site 4 & Site 5 \\
\hline \multicolumn{6}{|l|}{ Tabanidae } \\
\hline Chrysops spp. & $\mathrm{X}$ & $\mathrm{X}$ & & $\mathrm{X}$ & \\
\hline Chrysops aestuans Wulp & $\mathrm{X}$ & $\mathrm{X}$ & & $\mathrm{X}$ & \\
\hline Hybomitra cf. illota (Osten Sacken) & & & $\mathrm{X}$ & & \\
\hline \multicolumn{6}{|l|}{ Tipulidae } \\
\hline Helius spp. & $\mathrm{X}$ & & $\mathrm{X}$ & $\mathrm{X}$ & \\
\hline Prionocera spp. & & & & $\mathrm{X}$ & \\
\hline
\end{tabular}

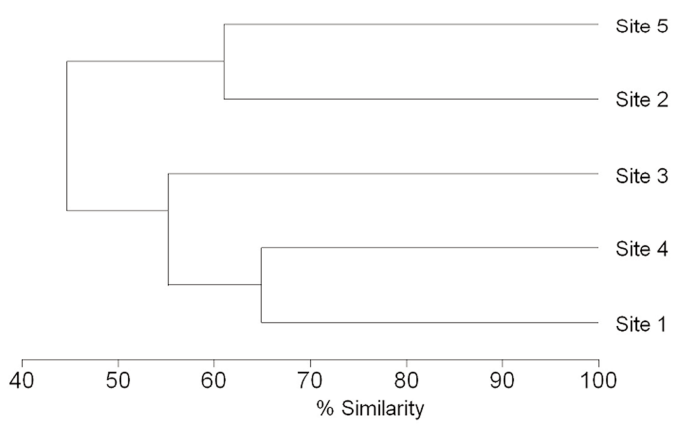

Fig. 2. Cluster analysis of site taxa presence/absence data.

with $100 \%$ alcohol and returned to the lab for processing. We took aerial sweep samples of the riparian vegetation to collect adult insects. These samples were preserved in $70 \%$ alcohol.

Representative specimens of each distinct macroinvertebrate type were sorted in the lab using the naked eye and a dissecting microscope at $6 \mathrm{X}$ from qualitative dip net samples, aerial sweep samples, and extra material from the composite proportional samples. These specimens were preserved in $70 \%$ alcohol, pinned, or mounted on microscope slides prior to identification. Voucher series were deposited in the Royal Saskatchewan Museum, Regina, and Saskatchewan Watershed Authority Invertebrate Survey, Saskatoon.

Composite proportional dip net samples were washed to remove any remaining silt using a 500-m sieve and then divided into $1 / 8$ or $1 / 16$ sample fractions using a piece of Plexiglas (Cuffney et al. 1993). Subsamples were randomly chosen and processed under a dissecting microscope at $6 \mathrm{X}$ until a count of 300 macroinvertebrates was attained or the entire sample had been processed. We identified macroinvertebrates to the lowest level possible and enumerated them. We assigned feeding group categories to each taxa based on the lit- erature (Pennak 1989, Clifford 1991, Merritt and Cummins 1996, Mandaville 2002). Data were then used to determine percent composition of each taxa (taxa individuals / total individuals * 100) and feeding group at the sites. Similarity cluster analysis was performed on taxonomic and feeding group data using a Multi-Variate Statistical Package (MVSP; Kovach 1999). The Weighted Pair Group Average Method (WPGMA) was used to account for fewer sampling dates at site 3 (Kovach 1999). Rarefaction was used to estimate the species richness accumulation curve (Colwell 2006).

Based on data in Mandaville (2002) and Barbour et al. (1999), we assigned pollution tolerance values to the taxa. Idaho values were used where possible, as they are the geographically closest set of values for northwest North America. If Idaho values were not available, average scores were calculated from other regions in the table. Water quality at the sites was assessed with the Modified Hilsenhoff Biotic Index (MHBI; Mandaville 2002), using the composite proportional samples. Because pollution tolerance values change for a particular stream over the course of a season, we used only April samples for organic pollution indication, based on the recommendations of Hilsenhoff (1988).

\section{Results}

Taxonomic Results

Here we record 294 macroinvertebrate taxa from 60 different families (Table 2) for the Pipestone Creek watershed. Non-insect macroinvertebrate taxa richness was dominated by mollusks; 1 limpet (Ferrissia rivularis), 14 snail taxa, and 3 mussels. Leeches were represented by at least 7 species belonging to 2 families: Erpobdellidae and Glossiphoniidae. Crustaceans collected include ostracods, the amphipods Gammarus lacustris and Hyallela azteca, and the crayfish Orconectes virilis. Water mites 


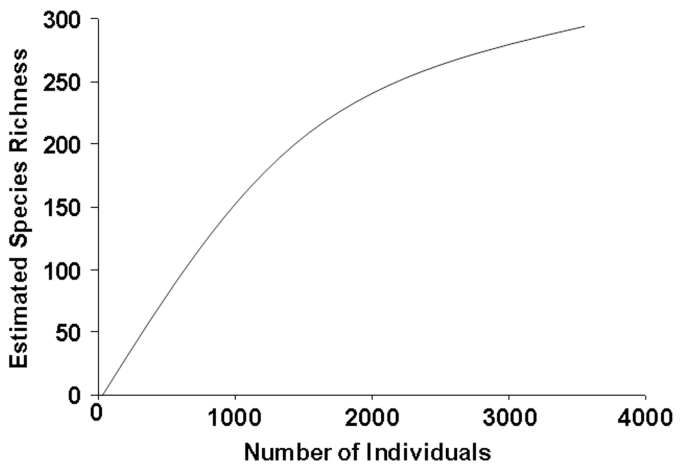

Fig. 3. Rarefaction estimate of species richness for benthic macroinvertebrate species sampled in the Pipestone River, Saskatchewan, Canada.

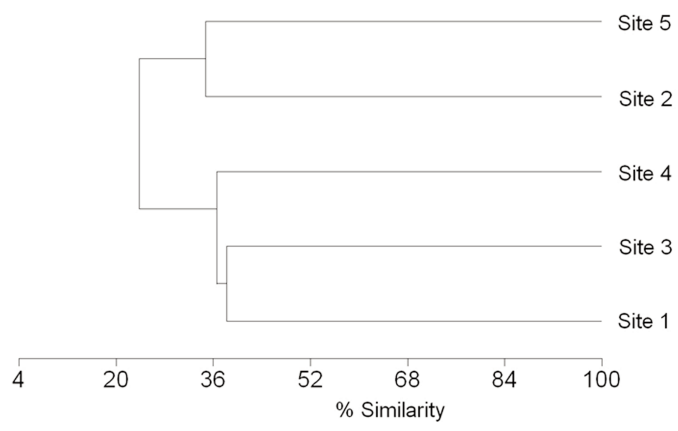

Fig. 4. Cluster analysis of taxa percent composition from proportional samples.

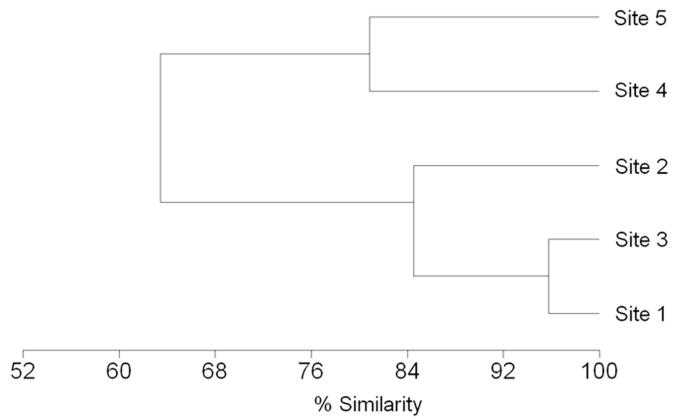

Fig. 5. Cluster analysis of feeding group percent data collected in proportional samples.

were collected at every site in low numbers. Freshwater sponges and bryozoans were also found at a few sites (Table 2).

Insects were the most diverse group found in the Pipestone watershed. We record 18 taxa representing 5 families of mayflies. The Baeti- dae were the most diverse with 9 genera/ species identified. The Odonata included 3 families of damselflies and 3 of dragonflies. A single Plecoptera species, Perlesta placida, was collected. Six families with at least 23 species of Hemiptera were collected, including the giant water bug Lethocerus americanus and the water scorpion Ranatra fusca. The most diverse hemipteran group found here was the Corixidae, with 14 species.

Six families of Trichoptera were recorded; the most diverse family was the Limnephilidae, with 13 species identified. At least 1 species of Lepidoptera was collected but the larvae could be identified only to Pyralidae. Saskatchewan's only species of Megaloptera, Sialis velveta, was also collected. At least 39 taxa of Coleoptera were collected. Dytiscidae was the most diverse family of beetles found, with 24 taxa (Table 2).

The Diptera was the most diverse insect order collected in this study. Eighty-five taxa belonging to 13 families were identified. Over half the taxa was comprised by family Chironomidae. Unfortunately, most of these could be identified only to genus, which probably underrepresented the actual diversity. Biting flies included at least 3 species of Ceratopogonidae, 2 species of both Culicidae and Tabanidae, and 4 Simuliidae species.

Cluster analysis of taxa presence/absence site data grouped sites 1 and 4 together as the sites with the most similar faunae, $64.9 \%$. Site 3 grouped with these 2 sites at $55.2 \%$ similarity. Sites 2 and 5 formed a distinct cluster at the $60.9 \%$ similarity level (Fig. 2).

Successive sampling dates increased the number of taxa recorded for each site and the entire study (Fig. 3); however, there is no obvious asymptote developed in the species accumulation curve (rarefaction; see Buddle et al. 2005) that would indicate taxonomic richness sampling saturation.

\section{Specific Taxonomic Composition}

Percent taxonomic composition of the proportional samples differed greatly among the sites. Cluster analysis of the data revealed that the 2 most similar sites, sites 1 and 3, had only $38.2 \%$ similarity (Fig. 4).

Cluster analysis of feeding group data from the proportional dip net samples separated the sites into 2 groups: sites 1, 2 , and 3 clustered at the $84.5 \%$ level; and sites 4 and 5 clustered slightly lower at the $80.9 \%$ level (Fig. 5). 
TABLE 3. Water quality characteristics based on the Modified Hilsenhoff Biotic Index (MHBI). Site values are in parentheses. From Mandaville 2002.

\begin{tabular}{lcl}
\hline Pipestone Creek site values & MHBI & Degree of organic pollution \\
\hline & $0.00-3.50$ & No apparent organic pollution \\
& $3.51-4.50$ & Possible slight organic pollution \\
Site $2(6.02)$ & $4.51-5.50$ & Some organic pollution \\
Site $1(6.93), 4(6.96), 5(6.87)$ & $5.51-6.50$ & Fairly significant organic pollution \\
Site $3(7.54)^{\mathrm{a}}$ & $6.51-7.50$ & Poor significant organic pollution \\
& $7.51-8.50$ & Very significant organic pollution \\
& $8.51-10.00$ & Severe organic pollution \\
\hline
\end{tabular}

aValues for May samples as no samples for April

Collectors accounted for over $50 \%$ of the macroinvertebrates collected at sites 1,2 , and 3. At sites 4 and 5 scrapers and grazers were the most important group, making up over $42 \%$ of the community.

Although sites 1, 2, and 3 were collector dominated, the faunal make-up at the sites was quite different. At site 1 the collectors primarily consisted of Chironomidae (45.7\%), particularly Tanytarsus (10.8\%). At site 2 the main collectors were pea clams (Pisidiidae; 20.8\%), especially Sphaerium (14.6\%), and chironomids $(15 \%)$. Collectors at site 3 included Chironomidae $(27 \%)$ and Ostracoda (17.1\%). The majority of scrapers and grazers at site 4 were $H$. azteca $(13.1 \%)$ and V. tricarinata (13\%). However, at site 5, H. azteca $(22.0 \%)$ and baetid mayflies $(15.1 \%)$ were the dominant scrapers and grazers.

The Modified Hilsenhoff Biotic Index, calculated from the proportional data, suggested that all sites were significantly polluted when compared with the water quality data (Table 3 ).

\section{Discussion}

Very little comprehensive community-based macroinvertebrate biodiversity research has been done on the waterways in southeastern Saskatchewan. This study recorded important Saskatchewan range extensions for 12 taxa of mayflies: Acerpenna pygmaea, Baetis brunneicolor, B. flavistriga, B. tricaudatus/brunneicolor, Callibaetis ferrugineus, Procloeon sp., Caenis amica, C. latipennis, Leucrocuta hebe, Stenacron interpunctatum, S. terminatum, and Leptophlebia sp. Most of these species were previously known from the more northern boreal forests of the province (Webb 2002). The presence of the stonefly Perlesta placida is also a range extension to the southeast corner of the province for this species (Dosdall and Lehm- kuhl 1979, Dosdall 1992) from limits at Cypress Hills to the west (about $600 \mathrm{~km}$ ), and north in the boreal forest (about $600 \mathrm{~km}$ ).

Trichoptera in Saskatchewan have not been well studied outside the boreal forests (Smith 1984). Twelve caddisflies identified in this study had range extensions in the southeast corner of Saskatchewan: Hydropsyche bifida, H. alternans, Ithytrichia clavata, Nectopsyche diarina, Oecetis immobilis, Limnephilus cf. janus, Triaenodes injustus, Limnephilus hyalinus, L. infernalis, L. labus, L. rhombicus, and Philarctus quaeris (Smith 1984, Nimmo 1987, Floyd 1995, Glover 1996, Wiggins 1998).

Because Pipestone Creek is linked to eastern waterways of Manitoba and Ontario, and ultimately the eastern extent of the Wisconsin Glacier, many more significant zoogeographical records are likely to be discovered in this area. The chironomid Zavreliella maromata was only recently reported in Saskatchewan near Regina (Parker and Glozier 2005). Its collection in the Pipestone Creek watershed may indicate the immigration path used to reach Saskatchewan from eastern Canada. This eastern connection also makes Pipestone Creek a potential route for "invasive species." If such species become established, they could alter the aquatic communities within the province.

The Pipestone Creek watershed also harbors significant populations of biting flies. Culicidae collected included Aedes sp. and Anopheles earlei. Numerous Ceratopogonidae larvae were collected, but it was impossible to reliably identify most of them below family level. Two species of Tabanidae were collected, Chrysops aestuans and Hybomitra cf. illota. The latter species attacks humans and livestock (Teskey 1990). At least 4 species of Simuliidae were also collected. All are known biters of both humans and livestock and can, in numbers, be significant pests (Fredeen 1981). 
Cluster analysis of the taxonomic presence/ absence data (Fig. 2) separates the sites into 2 clusters based on taxonomic preferences for water flow and vegetation. Sites 1, 3, and 4 are essentially lentic habitats and harbor many taxa, including lymnaeid snails, libellulid dragonflies, limnephilid caddisflies, and dytiscid beetles, which are most abundant and diverse in standing waters. In contrast, sites 2 and 5 are lotic habitats and contain macroinvertebrates more typical of flowing waters, such as the freshwater limpet (F. rivularis), heptageniid mayflies, blackflies, the single stonefly species, and hydropsychid caddisflies.

The accumulated taxa curve of the study has not yet begun to show a significant asymptote (Fig. 3), indicating that the taxonomic richness for Pipestone Creek has yet to be adequately estimated. The individual site totals represented $54 \%$ or less of the 294 taxa collected in the entire study. Single-date taxa counts account for less than $60 \%$ of the taxa collected and single-sample date and site combinations represent $27 \%$ or less of the total taxa collected. This is not surprising when one considers the heterogeneity of habitats present at each site in addition to the temporal faunal changes expected from seasonal life cycles. The low percent similarity of single-sample data in site combinations further emphasizes the necessity of broad spatial and temporal resolution when initiating watershedscale benthic macroinvertebrate biodiversity studies.

Pipestone Creek and its sampled tributaries appear to be impacted by organic pollutants based on values calculated from the Modified Hilsenhoff Biotic Index (Table 3). There are a multitude of possible sources of this pollution, including agricultural chemicals, sewage lagoon leakage, cattle corrals, etc. To identify the sources and determine if the pollution is due to a general source or point sources will require further research. Unfortunately, there is very little information on tolerance values and resulting pollution scales based on research in Saskatchewan. Tolerance values for the calculations were extrapolated from different geographic regions of North America, mainly Idaho (Barbour et al. 1999, Mandaville 2002). Such values need to be adjusted to suit each region (Mandaville 2002) and stream type. Furthermore, species-level tolerance values from these sources are incomplete, and generic and fam- ily-level values often have to be used. There is no indication that these supra-generic values are based on any species common to the present study. While this situation is definitely not ideal, it is currently all that is available and provides enough information to raise concerns about the water quality of the Pipestone Creek watershed, and it suggests that further monitoring of the system is necessary.

\section{ACKNOWLEDGMENTS}

Funding for research was provided by a grant from the Fish and Wildlife Development Fund through the Saskatchewan Watershed Authority. Thanks are extended to G. Ekert, T. Houderstad, W. Kemp, G. Rutledge, and J. van Eaton for allowing access to the collection sites. J. Halpin and V. Keeler provided field assistance. J. Halpin assisted in processing the samples. We thank D.A. Schulz and 2 anonymous reviewers for their insightful comments on earlier versions of this manuscript.

\section{Literature Cited}

Barbour, M.T., J. Gerritsen, B.D. Snyder, And J.B. StRIBLING. 1999. Bioassessent protocols for the use in streams and wadeable rivers: periphyton, benthic macroinvertebrates and fish. 2nd edition. EPA 841B-99-002, U.S. Environmental Protection Agency, Office of Water, Washington, DC. $306 \mathrm{pp}$.

Buddle, C.M., J. Beguin, E. Bolduc, A. Mercado, T.E. SaCketT, R.D. SElby, H. Varady-SZabo, and R.M. ZERAN. 2005. The importance and use of taxon sampling curves for comparative biodiversity research with forest arthropod assemblages. Canadian Entomologist 137:120-127.

Colwell, R.K. 2006. EstimateS: statistical estimation of species richness and shared species from samples [cited 1 March 2007]. Version 8.0.0. Available from: http://viceroy.eeb.uconn.edu/EstimateS

DosDALL, L.M. 1992. New records of Saskatchewan stoneflies (Plecoptera). Proceedings of the Entomological Society of Manitoba 48:14-31.

DosDall, L.M., AND D.M. LehmKuHL. 1979. Stoneflies (Plecoptera) of Saskatchewan. Quaestiones Entomologicae 15:3-116.

Clifford, H.F. 1991. Aquatic invertebrates of Alberta. University of Alberta Press, Edmonton, Alberta, Canada. 538 pp.

Cuffney, F., M.E. Gurtz, and M.R. Meador. 1993. Methods for collecting benthic invertebrate samples as part of the national water-quality assessment program. U.S. Geological Survey, Open-file Report 93406. $66 \mathrm{pp}$.

FLOYD, M.A. 1995. Larvae of the caddisfly genus Oecetis (Trichoptera: Leptoceridae) in statistics for field biology. 2nd edition. John Wiley \& Sons, Inc., New York. 259 pp. 
Fredeen, F.J.H. 1981. Keys to the black flies (Simuliidae) of the Saskatchewan River in Saskatchewan. Quaestiones Entomologicae 17:189-210.

GLOVER, J.B. 1996. Larve of the caddisfly genera Triaenodes and Ylodes (Trichoptera: Leptoceridae) in North America. Bulletin of the Ohio Biological Survey 11(2).

HilsenhofF, W.L. 1988. Seasonal correction factors for the biotic index. Great Lakes Entomologist 21:9-13.

KovacH, W.L. 1999. Multivariate statistical package. Version 3.1. Kovach Computing Services. Pentraeth, Wales, U.K.

MandaVille, S.M. 2002. Benthic macroinvertebrates in freshwaters - taxa tolerance values, metrics, and protocols. Project H-1, Soil and Water Conservation Society of Metro Halifax. xviii, 48 pp., Appendices A-B. 120 pp [total].

Merritt, R.W., and K.W. Cummins, editor. 1996. An introduction to the aquatic insects of North America. 3rd edition. Kendall Hunt Publishing Company, Dubuque, IA. 862 pp.

Nimmo, A.P. 1987. The adult Arctopsychidae and Hydropsychidae (Trichoptera) of Canada and adjacent United States. Quaestiones Entomologicae 23:1-189

Parker, D.W., AND N. GLOZIER. 2005. First record of the non-biting midge, Zavreliella marmorata (Wulp.) (Chironomidae: Diptera), from Saskatchewan. Blue Jay 63:200-202
Pennak, R.W. 1989. Freshwater invertebrates of the United States. 3rd edition. John Wiley \& Sons, Inc., New York. 628 pp.

Resh, V.H., and D.M. Rosenberg, editors. 1984. The ecology of aquatic insects. Praeger Scientific, New York. 625 pp.

SMITH, D.H. 1984. Systematics of Saskatchewan Trichoptera larvae with emphasis on species from boreal streams. Doctoral thesis, University of Saskatchewan, Saskatoon, Canada. 1302 pp.

Teskey, H.J. 1990. The insects and arachnids of Canada. Part 16, The horse flies and deer flies of Canada and Alaska. Research Branch, Agriculture Canada, Ottawa. Publication No. 1838. 381 pp.

Thore, J.H., AND A.P. Covich, EDITORs. 2001. Ecology and classification of North American freshwater invertebrates. 2nd edition. Academic Press, New York. $1056 \mathrm{pp}$.

WebB, J.M. 2002. The mayflies of Saskatchewan. Master's thesis, University of Saskatchewan, Saskatoon, Canada. $430 \mathrm{pp}$.

Wiggins, G.B. 1998. The caddisfly family Phryganeidae (Trichoptera). University of Toronto Press, Toronto, Canada. 306 pp.

Received 1 March 2007 Accepted 27 November 2007 